\title{
Mécanismes de dégradation et moyens de protection des câbles du génie civil
}

\section{Exemple des câbles de haubanage}

\author{
Lamine Dienga ${ }^{a}$, Virginie Périer, Laurent Gaillet et Christian Tessier
}

Laboratoire Central des Ponts et Chaussées, Division Métaux, Armatures et Câbles pour Ouvrages d'Art, Route de Bouaye, BP 4129, 44341 Bouguenais Cedex, France

Reçu le 28 août 2008, accepté le 9 avril 2009

\begin{abstract}
Résumé - Les câbles actuellement utilisés en génie civil, notamment sur les ponts à haubans ou suspendus, sont constitués de fils d'acier cylindriques de diamètre compris entre 3 et $7 \mathrm{~mm}$. Ils sont en acier non ou peu allié de composition proche de l'eutectoïde et sont obtenus par tréfilage à froid. Ils ont une résistance à la traction élevée (supérieure à $1900 \mathrm{MPa}$ ) et une faible ductilité. De par leur constitution, les câbles présentent une bonne robustesse en traction et une faible rigidité à la flexion. Cependant, les études conduites ces dernières années ont montré que les principales causes de détérioration des câbles sont liées à la corrosion et aux frottements interfilaires induits par la fatigue. Ces mécanismes de dégradation des câbles sont décrits dans cet article. Les différents moyens de protection des câbles du génie civil ont été initialement développés pour lutter contre la corrosion. Ils se présentent sous la forme de revêtements de type peinture, galvanisation, galfanisation, associées à une lubrification. Certains peuvent cependant avoir aussi des effets sur la fatigue. Les résultats expérimentaux et numériques obtenus sur fils unitaires, présentés dans cet article, montrent clairement l'effet bénéfique de la galvanisation et de la lubrification en termes de durée de vie en fretting fatigue. Cependant, les mécanismes de dégradation dus au fretting fatigue en présence de corrosion ne sont pas totalement connus et font l'objet actuellement d'une étude approfondie.
\end{abstract}

Mots clés : Câble métallique / fatigue / fretting / corrosion

Abstract - Degradation mechanisms and protective methods of civil engineering cables example of stay cables. Cables currently used in civil engineering, including cable-stayed bridges or suspension bridges, are made of cylindrical steel wires with a diameter between 3 and $7 \mathrm{~mm}$. They are unalloyed or low alloyed steel whose composition is near eutectoid and are obtained by cold drawing. They have a high resistance in traction (more than $1900 \mathrm{MPa}$ ) and a low ductility. By their constitution, cables have high tensile strength and low bending stiffness. However, the studies conducted in recent years have shown that the main causes of cables deterioration are due to corrosion and to inter-wires frictions induced by fatigue. These cables degradation mechanisms are described in this paper. Different methods of protecting civil engineering cables were originally developed against corrosion, such as metal coatings (galvanization, galfanization), and lubrication. These protections can also have effects on fatigue. The experimental and numerical results obtained from single wires and presented in this paper, show obviously the beneficial effect of galvanizing and lubrication in terms of fretting fatigue lifetime. However, the mechanisms of degradation due to fretting fatigue in the presence of corrosion are not fully known and are currently the subject of a thorough study.

Key words: Metallic cable / fatigue / fretting / corrosion

\footnotetext{
${ }^{a}$ Auteur pour correspondance : lamine.dieng@lcpc.fr
} 


\section{Nomenclature}

\begin{tabular}{|ll|}
\hline$R e$ & $(\mathrm{MPa})$ Limite élastique \\
$\sigma_{\mathrm{R}}$ & $(\mathrm{MPa})$ Contrainte ultime \\
$\sigma_{\mathrm{Rp} 0,02}$ & $(\mathrm{MPa})$ Limite élastique pour un offset $=0,02 \%$ \\
$\mathrm{~T} 15,7$ & Torons 7 fils de diamètres $15,7 \mathrm{~mm}$ \\
$\varepsilon$ & $(\mathrm{m} / \mathrm{m})$ Déformation \\
$\Delta \sigma$ & $(\mathrm{MPa})$ Amplitude de variation de contrainte \\
$E$ & $(\mathrm{MPa})$ Module de Young \\
$F_{\mathrm{c}}$ & $(\mathrm{N})$ Force de contact \\
$\mathrm{A}$ & $\left(\mathrm{m}^{2}\right)$ Section du fil \\
$\nu$ & Coefficient de Poisson \\
$\sigma_{y}$ & $(\mathrm{MPa})$ Limite élastique \\
$\mu$ & Coefficient de frottement \\
$\Delta \tau$ & $(\mathrm{MPa})$ Amplitude de la contrainte \\
& de cisaillement \\
$P h$ & $(\mathrm{MPa})$ Pression hydrostatique \\
$a, b$ & Caractéristiques du matériau \\
$\sigma_{\mathrm{I}}, \sigma_{\mathrm{II}}, \sigma_{\mathrm{III}}$ & $(\mathrm{MPa})$ Contraintes principales \\
\hline
\end{tabular}

\section{Introduction}

Suivant leur domaine d'utilisation et les différentes époques, il existe une grande variété de types de câbles et leur utilisation s'est répandue depuis le $19^{\mathrm{e}}$ siècle dans de nombreux domaines industriels : on peut citer l'industrie minière qui fut l'une des premières à utiliser les câbles d'acier pour équiper les mines, mais aussi les systèmes de levage, les câbles de lignes électriques, les ascenseurs, les caténaires, les ancrages de plates-formes offshore, les ponts suspendus, les ponts à haubans ou encore les structures précontraintes. Dans le génie civil, les câbles métalliques sont présents dans de nombreux ouvrages comme les ponts en béton précontraints, les ponts suspendus, les ponts à haubans ou les téléphériques. Ce sont des éléments de structure essentiels qui sont soumis à des chargements variables liés aux sollicitations de service et aux sollicitations climatiques. Les propriétés mécaniques et rhéologiques des câbles en acier à haute résistance ont fait l'objet d'études expérimentales et théoriques assez complètes.

Les câbles des ouvrages d'art sont principalement (en France) des câbles monotoron multicouches à pas croisés et des câbles à multitorons ( 7 fils) parallèles. Pour ce type de câble, le comportement en fatigue est essentiellement lié aux effets des contacts entre fils et aux effets des contacts des fils avec les éléments d'ancrage (ou de déviation). Chaplin (1995) exprime les constatations générales qui suivent [1] :

- les contacts sont à l'origine d'une réduction importante de la limite d'endurance des assemblages de fils (câbles) par rapport à celle du fil isolé;

- les surfaces de contact sont le siège de dégradations par usure.

La connaissance du comportement à la fatigue du fil est insuffisante pour prédire directement celui du câble. En effet la résistance à la fatigue d'un fil isolé est supérieure à celle d'un même fil incorporé dans un câble (Fig. 1) [3] .

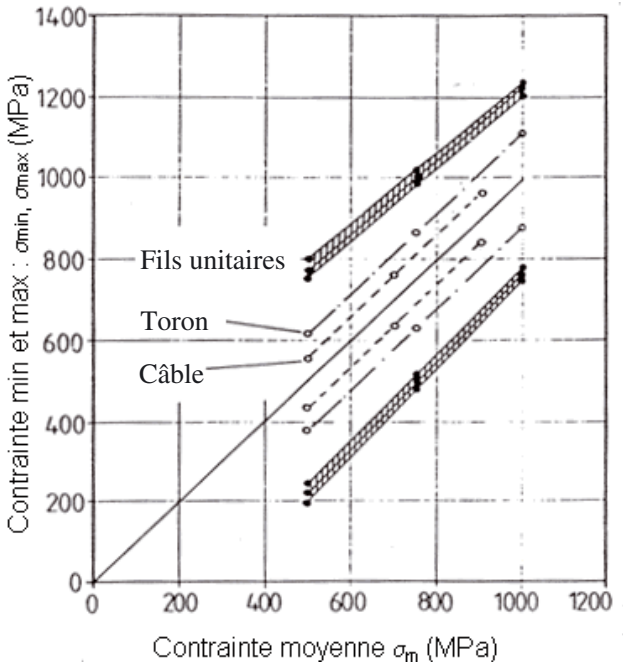

Fig. 1. Diagramme de Becker [3].

Ces généralités sont les seules communes à l'ensemble des câbles. Les fonctionnements spécifiques à chaque application doivent être pris en compte pour l'étude du comportement en fatigue : fonctionnement à durée de vie limitée (câbles de levage), fonctionnement dans le domaine d'endurance (câbles de ponts), sollicitations en traction ondulée (pont, câble de transport), sollicitations de flexion à courbure imposée (passage sur poulies), sollicitations de flexion libre (câbles de pont), sollicitations en torsion (câbles de transport aérien), ...

Les éléments qui vont être développés concernent donc l'utilisation particulière des câbles de haubans tendus aux charges moyennes voisines de $30 \%$ de la charge de rupture, soumis à des petites déformations cycliques au sens de la mécanique et travaillant dans le domaine proche de la limite d'endurance infinie.

\section{Définition des câbles de haubanage}

Les câbles de haubanage permettent de relier un pylône au tablier en différents points. Ils ont pour principale fonction de transmettre les sollicitations du tablier au pylône. Ils peuvent être disposés soit unitairement, soit en nappes, soit en faisceaux. Leurs caractéristiques d'usage sont la résistance à la rupture et la raideur axiale (module d'élasticité apparent). Ces caractéristiques découlent des caractéristiques du fil de base : fil d'acier tréfilé clair ou revêtu de zinc par galvanisation à chaud $(R e \approx 1550$ à $1860 \mathrm{MPa}$ ), de la géométrie de toronnage (angle de toronnage de chaque couche, diamètre des fils) et de la géométrie de câblage : câble toronné multicouche, câble à fils parallèles, câble à torons ( 7 fils) parallèles.

\subsection{Processus d'élaboration et caractéristiques du fil d'acier}

Les fils pour câbles utilisés dans les ouvrages d'art sont en acier non (ou faiblement) allié avec une teneur en 


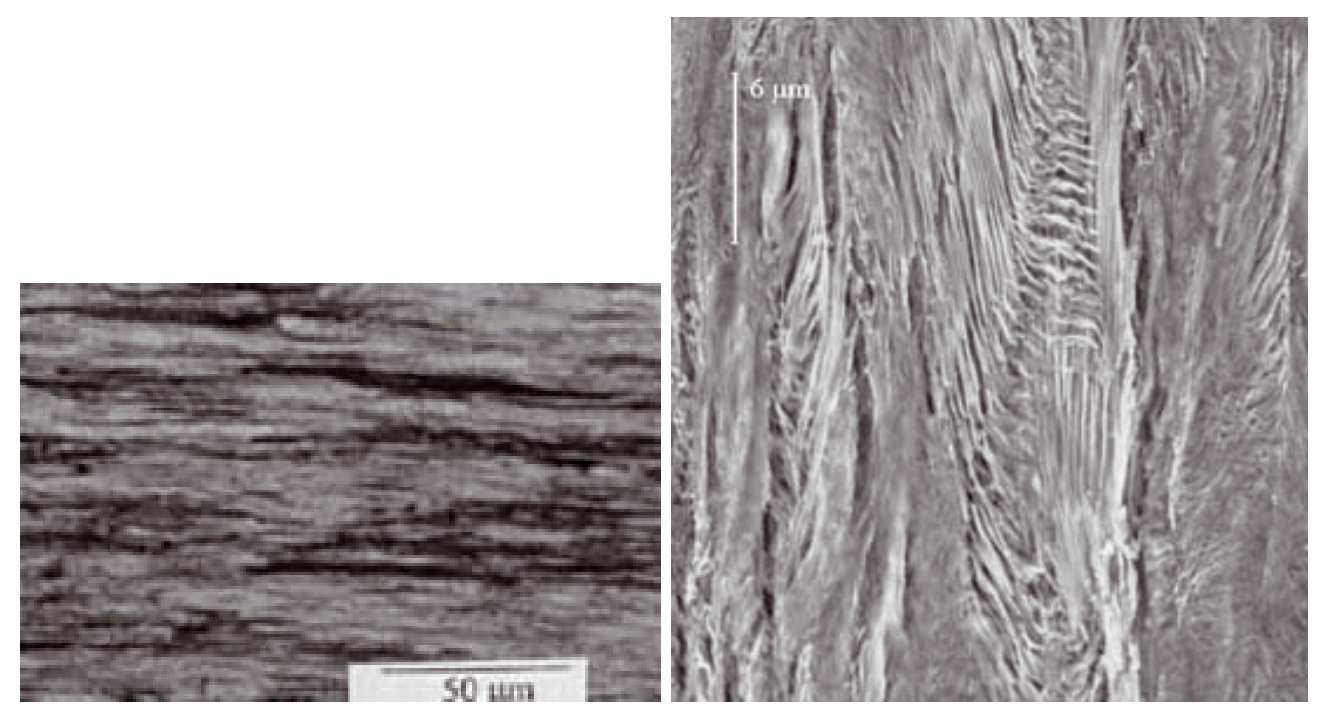

(a)

(b)

Fig. 2. Microstructure d'un fil d'acier tréfilé montrant la perlite et la texture allongée des grains [4], (a) observation optique, (b) observation au MEB.

Tableau 1. Caractéristiques mécaniques d'un fil et d'un toron.

\begin{tabular}{ccccc}
\hline & $E(\mathrm{MPa})$ & $\sigma_{\mathrm{R}}(\mathrm{MPa})$ & $\sigma_{\mathrm{Rp} 0,02}(\mathrm{MPa})$ & $\varepsilon(\%)$ \\
\hline fil & 202000 & 2020 & 1750 & 5,5 \\
toron & 193100 & 1940 & 1480 & 5,2 \\
\hline
\end{tabular}

carbone voisine de $0,7 \%$ et une teneur en manganèse proche de $1 \%$. Les fils sont obtenus à partir de filsmachine d'un diamètre voisin de $10 \mathrm{~mm}$, dont la forme cylindrique est obtenue par laminage à chaud. Les fils pour les câbles ont un diamètre usuellement compris entre 3 et $6 \mathrm{~mm}$. Pour fabriquer un fil, la section du fil machine est réduite soit par tréfilage à froid, soit par laminage à chaud. Cette dernière opération est suivie d'un refroidissement rapide (trempe).

Pendant une opération de tréfilage [4], la réduction de section de l'acier est effectuée progressivement, par passage du fil à travers des filières de diamètres décroissants jusqu'à obtenir le diamètre de fil et les caractéristiques mécaniques souhaités. Les déformations plastiques hétérogènes imposées lors du tréfilage engendrent, par le retour élastique du matériau en sortie des filières, des contraintes résiduelles. Ces contraintes sont de traction en surface et de compression en cœur. Le tréfilage augmente la résistance mécanique des fils par écrouissage et orientation des grains, ce qui donne au matériau une structure perlitique fortement anisotropique (Fig. 2). Le tableau 1 donne les caractéristiques mécaniques d'un fil d'acier de 5,4 mm de diamètre.

\subsection{Le toronnage}

Le toron est l'élément câblé le plus simple, il est constitué d'une ou plusieurs couches de fils enroulés en

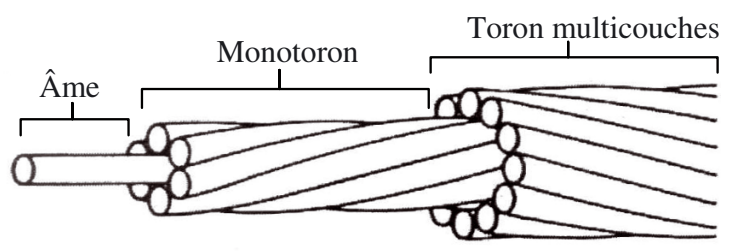

Fig. 3. Toron ordinaire de $19(1+6+12)$ fils.

hélice autour d'un fil droit central appelé âme (Fig. 3). On distingue deux types de torons : les torons ordinaires et les torons à fils parallèles [5].

Les torons ordinaires sont formés de couches successives comportant des fils de même diamètre mais en nombres différents. Chacune de ces couches est enroulée avec un pas d'hélice différent et lorsqu'on passe d'une couche à une autre, le nombre de fils hélicoïdaux est augmenté de 6 (Fig. 3). Ce sont les torons les plus utilisés en ouvrage d'art.

Les torons à fils parallèles, sont quant à eux constitués de fils de diamètres différents soit d'une couche à l'autre dans le cas du toronnage « Seale» (Fig. 4a), soit dans une même couche dans le cas d'un toronnage « Warrington » (Fig. 4b). De cette façon il est possible d'avoir tous les fils au même pas d'hélice. Le premier avantage de ce type de toronnage est que la fabrication peut alors être réalisée en une seule passe. Le deuxième avantage est qu'il permet d'obtenir des contacts linéiques entre fils au lieu de contacts ponctuels dans le cas du toronnage ordinaire. On diminue ainsi la pression de contact interfilaire et favorise le glissement relatif. Une combinaison de ces deux types de toronnage existe et est appelé toronnage WarringtonSeale (Fig. 4c) [2]. 

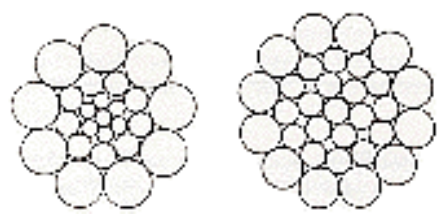

(a)

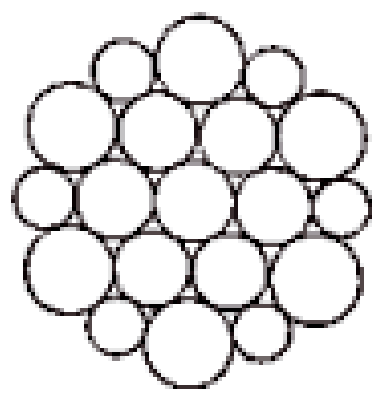

(b)
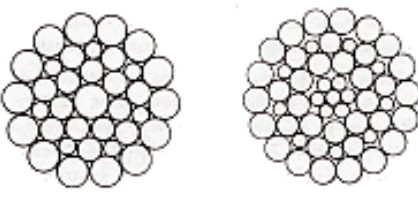

(c)

Fig. 4. Sections des différents types de torons à fils parallèles, (a) torons Seale de $25(1+6+9+9)$ et 31 (1+6+12+12) fils, (b) toron Warrington de $19(1+6+6,6)$, (c) torons Warrington-Seale de $36(1+7+7,7+14)$ et $52(1+6+9+9,9+18)$ fils.

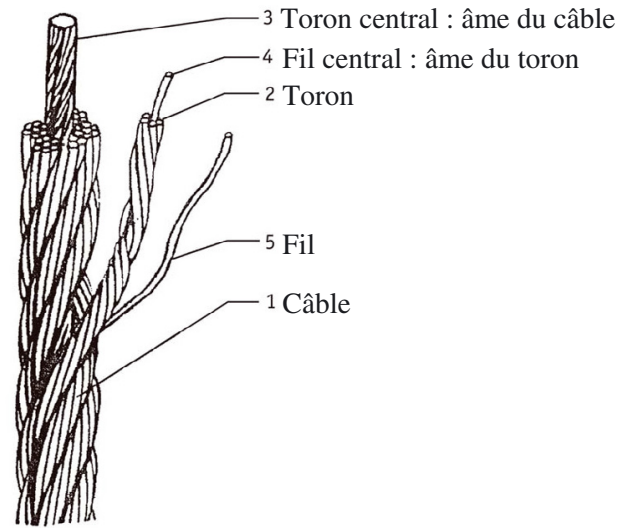

Fig. 5. Structure d'un câble à torons torsadés.

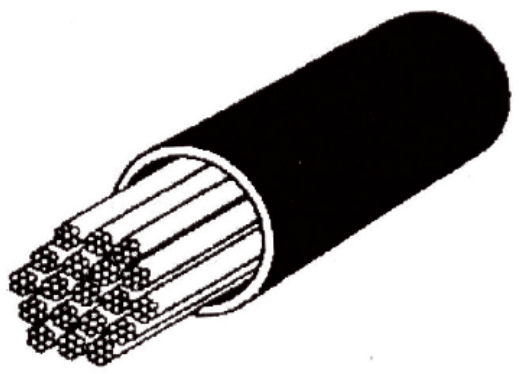

Fig. 6. Câble à torons parallèles.

\subsection{Le câblage}

Les torons peuvent être utilisés seuls ou comme éléments constitutifs des câbles. Il existe ainsi plusieurs types de câbles qui dépendent à la fois de la composition des torons utilisés et leur configuration géométrique dans le câble $[6,7]$. On distingue ainsi :

- des câbles à torons torsadés de géométrie très complexe; les torons sont disposés en hélice en une ou plusieurs couches sur âme centrale dont la composition varie : elle peut être en textile naturel ou synthétique, ou encore un toron ou un câble en acier (voir Fig. 5);
- des câbles à torons parallèles qui ont la particularité d'être constitués d'un ensemble de torons tendus en parallèle et ancrés individuellement dans une plaque par coincement de clavettes dans un trou conique;

- des câbles à fils parallèles, entourés d'une gaine métallique, qui sont utilisés dans les ponts suspendus.

L'effort global de traction $P$ selon l'axe du câble est réparti entre les différents fils. Pour déterminer le module apparent du câble, il faut tenir compte de plusieurs paramètres liés à la nature du câble tels que la rotation des couches externes, la contraction latérale du câble et les frottements interfilaires produits par la mise en tension. La valeur du module apparent dépend donc à la fois des caractéristiques mécaniques des fils constitutifs et de leur configuration géométrique. Il est de l'ordre de $210000 \mathrm{MPa}$ pour les câbles à fils parallèles, $200000 \mathrm{MPa}$ pour les câbles à torons parallèles, 160000 à $170000 \mathrm{MPa}$ pour les câbles multicouches.

Les câbles de haubanage se distinguent également par les types d'ancrage utilisés. La figure 7 présente l'ancrage par culottage au zinc ou à la résine. Ce type d'ancrage entraîne généralement la suppression des contacts entre fils dans l'encastrement et, par conséquent, diminue le risque de rupture par fatigue en traction ondulée $[1,8,9]$ si le contact glissant entre câble et culot d'ancrage est évité.

La figure 8 montre l'ancrage d'un toron unitaire par clavette (mors coniques). Pour ce type d'ancrage, la géométrie des stries et la géométrie relative des cônes mâle et femelle modifient de façon importante la limite d'endurance en traction ondulée.

Les détails des systèmes d'ancrage et des principes de fonctionnement sont proposés par [10]. Les systèmes de suspension et de haubanage sont, par ailleurs, décrits dans le guide des ponts suspendus [11] et dans l'ouvrage «Ponts à Haubans » de [12].

\section{Mécanismes de dégradation et moyens de protections}

Les haubans ont divers composants pouvant être affectés à long terme par les effets de l'environnement, 


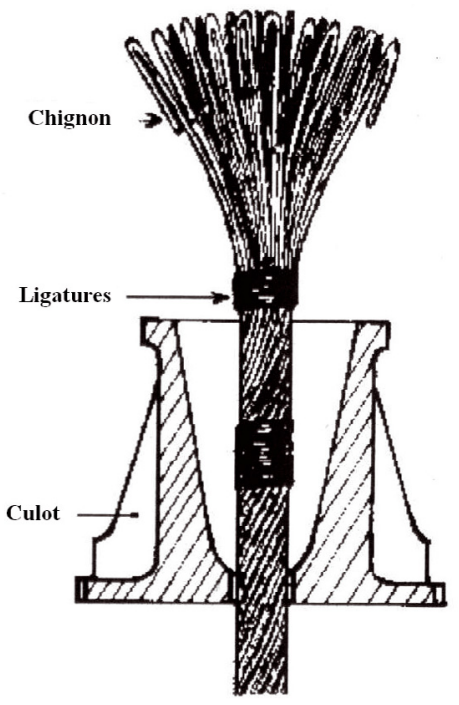

Fig. 7. Culot d'ancrage.

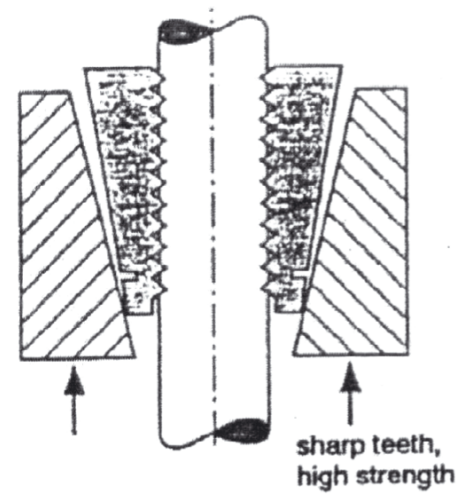

Fig. 8. Ancrage par clavette.

des sollicitations, ou du vieillissement naturel des matériaux : ancrages, câbles, produits de protection, tubes guides, amortisseurs, peintures ou gaines. Ces dégradations évoluent et peuvent conduire à quelques désordres mineurs ou plus sérieux qui guident l'évolution des technologies.

L'expérience est ancienne sur les câbles clos ou les câbles multicouches et a permis de mettre en évidence quelques mécanismes de dégradation qui sont d'ordre chimique (corrosion) et/ou mécanique (fatigue-fatigue de contact). Ces mécanismes ont également été rencontrés sur des câbles multifils ou multitorons parallèles.

\subsection{Problèmes liés aux phénomènes de corrosion}

Les phénomènes de corrosion n'apparaissent, en général, qu'après des durées de vie respectables car la connaissance de la sensibilité des aciers à la corrosion a toujours conduit à la mise en place de systèmes de protection interne et externe. Les analyses de rupture en service montrent que les ruptures de fils sont très

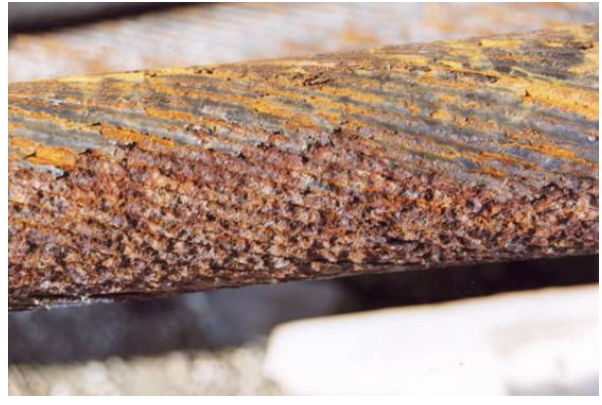

Fig. 9. Zone de dissolution sur un multicouche.

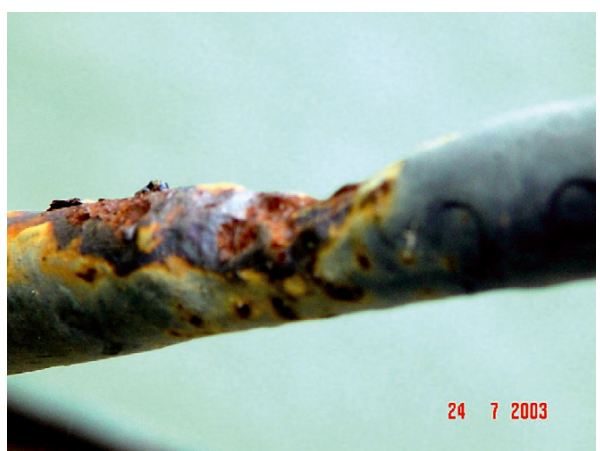

Fig. 10. Zone de corrosion localisée sur un fil.

souvent groupées dans les zones particulières (points bas de l'ouvrage). Liée à des défauts d'étanchéité, la circulation d'eau entraîne une corrosion dans ces zones.

\section{- La corrosion généralisée ou dissolution}

Elle se manifeste par la diminution de section des fils allant jusqu'à leur rupture (Fig. 9). Un effet induit est la perte d'étanchéité de la couche de fils atteints. L'origine de cette corrosion est généralement le non renouvellement de la protection, la rupture locale de la protection et le vieillissement de la protection interne. L'utilisation de fils galvanisés est un élément permettant de retarder l'apparition de ces phénomènes.

\section{- La corrosion localisée}

Ce phénomène correspond à plusieurs formes de corrosion dont les principales, sont la formation de piqûres (ou de crevasses), le couplage galvanique et la corrosion sous contrainte. La corrosion par piqûres ou cratères de corrosion a pour effet de fortement localiser l'endommagement du câble (Fig. 10). Sa cinétique de dégradation est d'autant plus rapide que le défaut est localisé.

La corrosion galvanique se produit en présence de deux matériaux différents du point de vue électrochimique ou entre deux zones rendues différentes au sein du même matériau par les conditions environnementales (aération différentielle,...) (Fig. 11).

La forme de corrosion localisée la plus dangereuse est la corrosion sous contrainte (CSC). En effet, il n'y a la plupart du temps aucun signe annonciateur avant la rupture brutale du câble hormis une faible corrosion en surface (Figs. 12 et 13). Son mécanisme repose sur la formation 


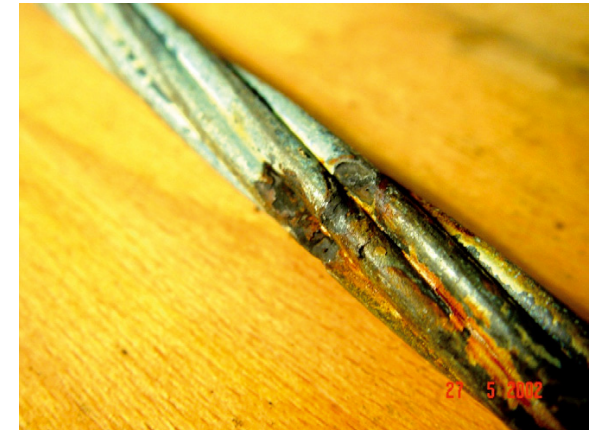

Fig. 11. Corrosion galvanique d'un toron galvanisé par couplage galvanisation/acier.

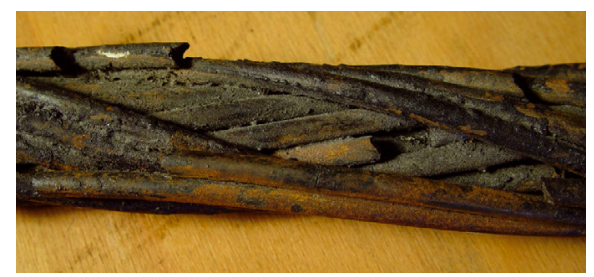

Fig. 12. Ruptures multiples par CSC sur un câble multicouche.

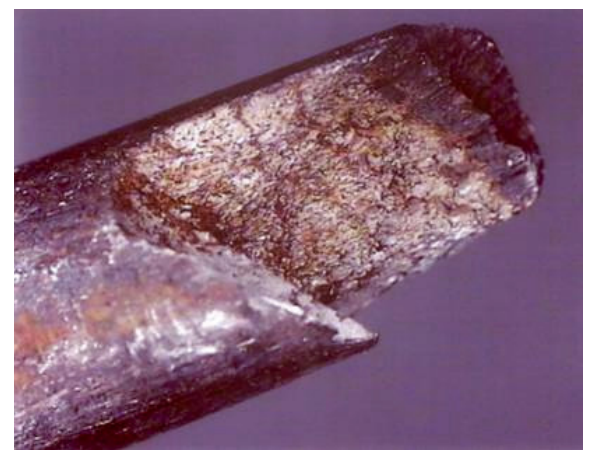

Fig. 13. Faciès de rupture semi-fragile sans réduction de section.

puis la propagation d'une fissuration dans le matériau. Les sites d'amorçage de ces fissures sont les défauts locaux (piqûre, crevasse) en surface du câble. Elle résulte de l'association d'une surconcentration locale en contrainte et d'un milieu corrosif (eau + produits de ruissellement) car la contrainte d'exploitation appliquée est relativement faible (inférieure au seuil de corrosion sous contrainte). L'utilisation d'une galvanisation, d'une protection interne pérenne et le maintien de l'étanchéité des câbles sont des conditions qui permettent d'en réduire l'occurrence.

\subsection{Fatigue et fretting-fatigue}

\section{- Étude expérimentale}

Des phénomènes de fatigue en traction pure n'ont été observés que sur des haubans provisoires et des haubans multifils. En laboratoire, les ruptures pour les câbles multitorons parallèles ou multifils apparaissent au niveau des ancrages (clavettes).
Des déformations de flexion (flexion circulaire ou flexion libre) provoquent entre fils de couches successives et entre torons parallèles des glissements qui conduisent à la fissuration et à la rupture par fatigue si :

- les amplitudes de flexion (variation de courbure) sont « fortes »;

- les contacts sont mal ou plus lubrifiés ;

- les revêtements métalliques sont usés.

D'autres contacts entre câbles et pièces de la structure peuvent provoquer ce même phénomène associant variation de contrainte globale (traction flexion) et contrainte de frottement de contact.

Pour étudier ce phénomène dit de fretting-fatigue, un dispositif d'essais de fretting-fatigue (Fig. 14) a été mis en place pour reproduire les conditions de sollicitations des fils de câbles à haubans. Il est constitué d'un fil d'acier tréfilé que l'on soumet à un chargement de fatigue en traction ondulée, et une amplitude de variation de contraintes $\Delta \sigma$. Ce fil est également soumis à des actions de contact en petits débattements puisqu'il est en contact avec quatre patins maintenus perpendiculaires au fil dans une pince. Une force de contact $F_{\mathrm{c}}$ est appliquée sur la pince pour reproduire la force de contact interfilaire. L'amplitude $T$ des forces tangentielles de contact est évaluée grâce à deux jauges de déformation qui permettent d'obtenir les déformations entre les patins et en dehors des patins, elle se calcule à partir de la relation suivante :

$$
T=\frac{E A}{2}\left(\varepsilon_{\mathrm{ext}}-\varepsilon_{\mathrm{int}}\right)
$$

Ce dispositif d'essai a été développé par [13] pour étudier l'effet de la lubrification sur le comportement en frettingfatigue des fils d'acier. Il a ensuite été utilisé par [15] pour évaluer l'effet de la galvanisation sur la fatigue de contact des fils.

Ces études ont montré les effets bénéfiques de la lubrification et de la galvanisation sur la durée de vie en fretting-fatigue des fils d'acier tréfilé (Fig. 15). Ce dispositif a été adapté pour étudier actuellement l'interaction entre le fretting-fatigue et la corrosion [16].

\section{- Étude numérique}

Ce dispositif expérimental de fretting-fatigue est à l'origine d'une modélisation numérique 2D du contact entre deux fils croisés à $90^{\circ}$. Pour des raisons de symétrie, seuls un demi-patin et un demi-fil ont été modélisés. Dans ce modèle par éléments-finis, le comportement du matériau est isotrope et élastoplastique parfait. Le patin et le fil ont été maillés à l'aide d'éléments triangulaires à trois nœuds, en prenant le soin de mailler plus finement la zone de contact (Fig. 16).

Une force de contact $F_{\mathrm{c}}$ de $200 \mathrm{~N}$ a été appliquée sur le patin sous la forme d'une pression linéique. Le fil a été soumis à une traction initiale $\sigma_{0}$ de $600 \mathrm{MPa}$, et à son extrémité droite, la contrainte varie de $\pm \Delta \sigma / 2$. Les caractéristiques du fil d'acier tréfilé sont rappelées dans le tableau 2. Ces modèles permettent d'observer la répartition des différentes composantes des contraintes au 

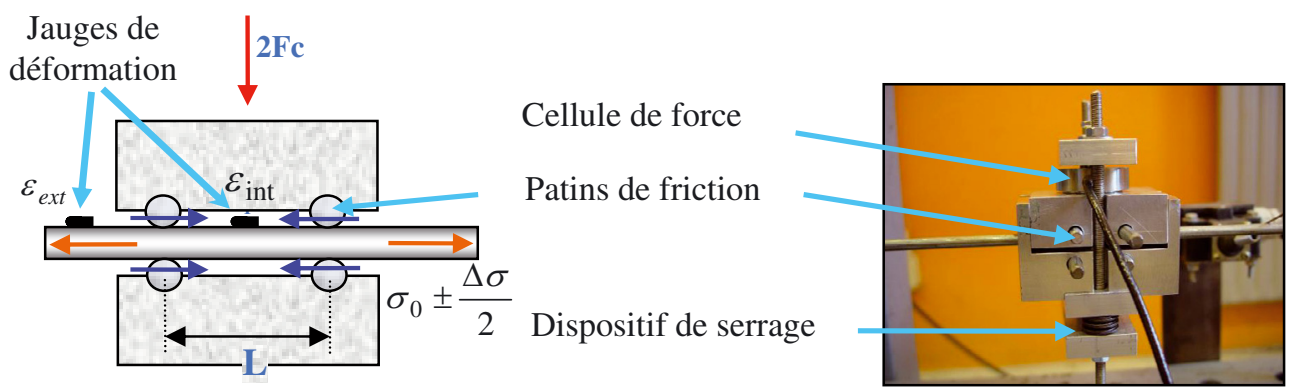

Fig. 14. Dispositif expérimental de fretting-fatigue.

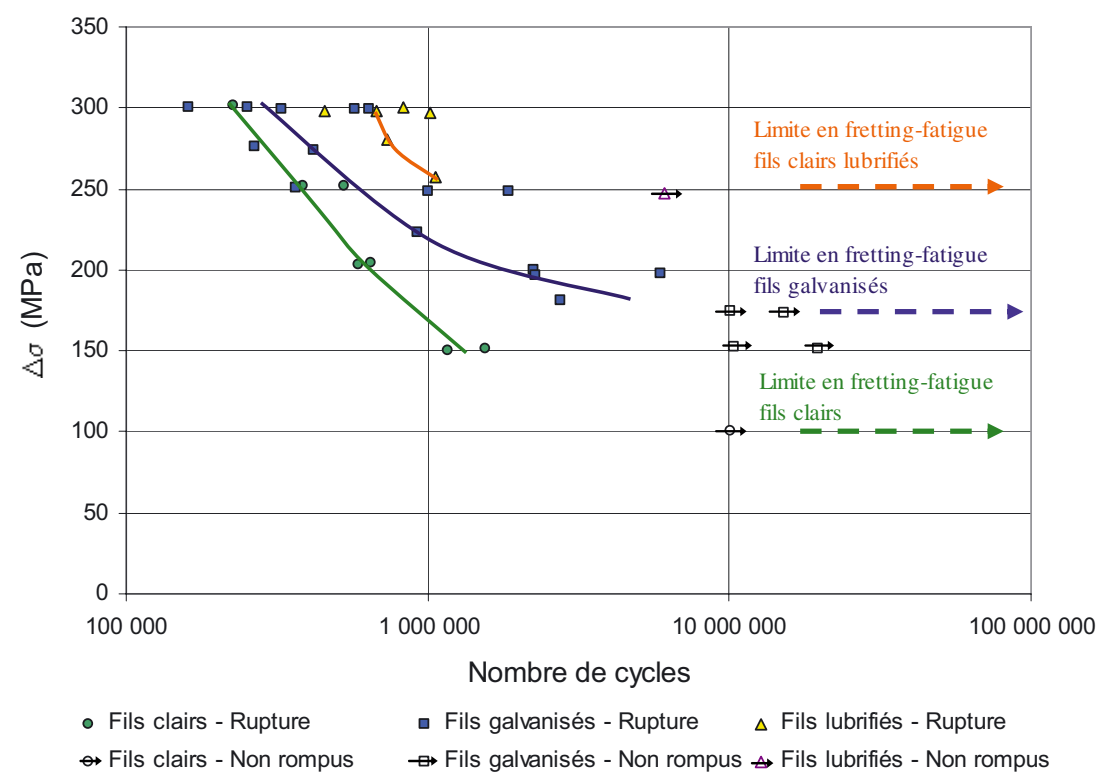

Fig. 15. Diagramme de Woehler - durée de vie en fretting-fatigue des contacts clairs, galvanisés et lubrifiés.

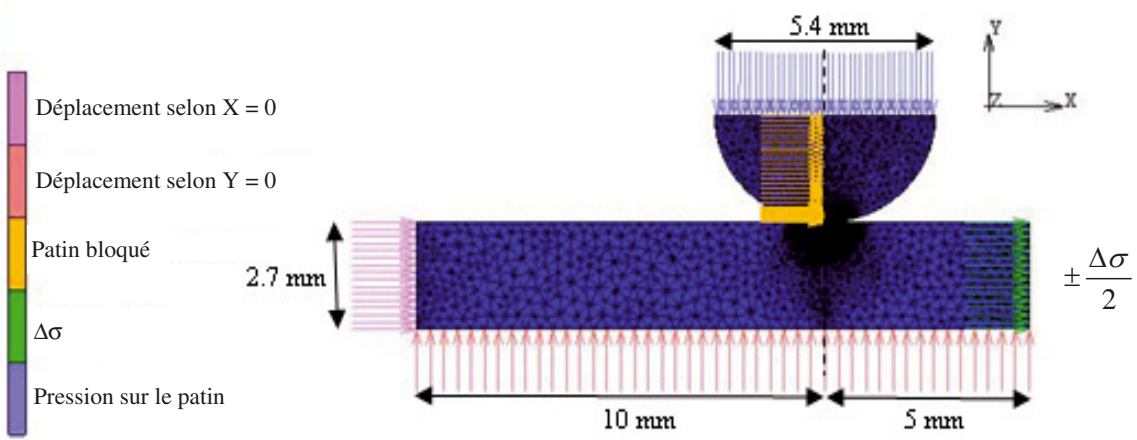

Fig. 16. Modèle 2D.

Tableau 2. Caractéristiques mécaniques introduites dans le modèle.

\begin{tabular}{ccc}
\hline$E(\mathrm{MPa})$ & $\nu$ & $\sigma_{y}(\mathrm{MPa})$ \\
\hline 210000 & 0,3 & 1500 \\
\hline
\end{tabular}

sein du fil et d'étudier l'influence du coefficient de frottement sur le niveau de contraintes en surface du fil. En effet, différents coefficients de frottement, allant de l'absence de frottement $(\mu=0)$ jusqu'au contact presqu'adhérant $(\mu=0,8)$ en passant par un contact lubrifié $(\mu=0,3)$, ont été introduits.

Des critères de fatigue ont également été utilisés afin de pouvoir distinguer un domaine sain d'un domaine d'initiation potentielle des fissures. Dans cette étude, le critère de Crossland [17] a été utilisé, il s'écrit sous la forme :

$$
\Delta \tau+b P h<a
$$




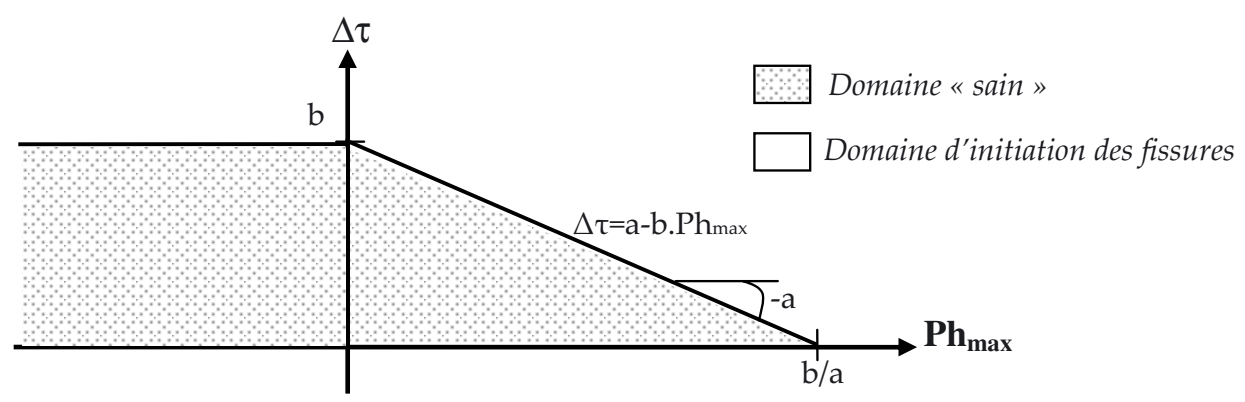

Fig. 17. Représentation du critère de fatigue de Crossland.

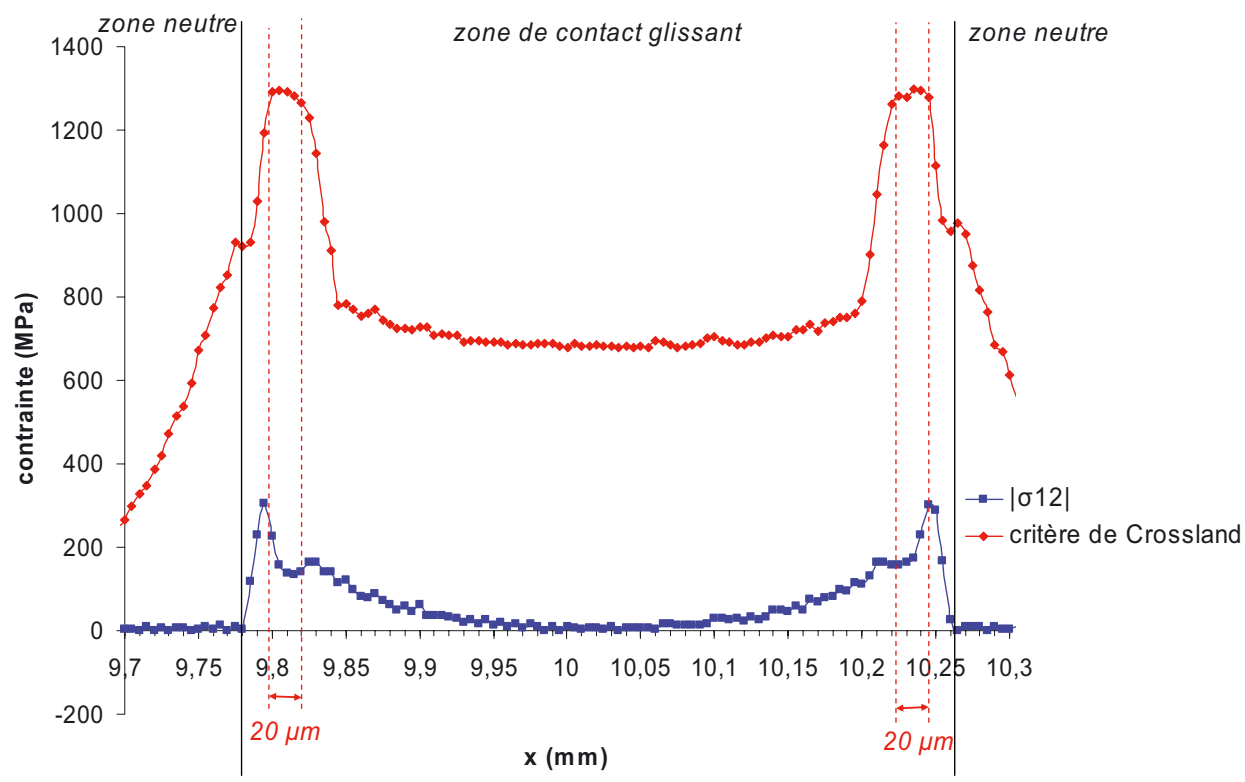

Fig. 18. Vérification du critère de Crossland à la surface du fil pour $\mu=0$ et $\Delta \sigma / 2=100 \mathrm{MPa}$, sous charge.

Ce type de critère permet de prendre en compte la pression hydrostatique $P h$

$$
P h=\frac{1}{3} \operatorname{Tr}(\sigma)
$$

ainsi que l'amplitude de la contrainte de cisaillement $\Delta \tau$

$$
\Delta \tau=\max \left[\left|\sigma_{I}-\sigma_{I I}\right| ;\left|\sigma_{I I}-\sigma_{I I I}\right| ;\left|\sigma_{I}-\sigma_{I I I}\right|\right]
$$

Les constantes $a$ et $b$ sont des caractéristiques du matériau qui peuvent être identifiées à partir d'essais de fatigue en torsion et/ou en flexion alternées.

Le critère de Crossland sert à déterminer les zones saines et les zones potentielles d'initiation de fissure (Fig. 17). Lorsque l'inéquation précédente est vérifiée, on se trouve dans le domaine d'endurance, sinon il s'agit d'une zone potentielle d'apparition des fissures, notamment aux endroits où ce critère est maximal.

Cette étude a consisté à observer le critère de Crossland à la surface du fil au niveau de la zone de contact, en fonction du coefficient de frottement appliqué et de la variation de contraintes appliquée. Les figures 18 et 19 représentent les contraintes $\left|\sigma_{12}\right|, \mu^{*}\left|\sigma_{22}\right|$ et le critère de Crossland

$$
\left(\begin{array}{l}
\Delta \tau+b P h_{\max }-a \quad \text { si } P h_{\max }>0 \\
\Delta \tau-a \quad \text { si } P h_{\max } \leqslant 0
\end{array}\right)
$$

calculés à la surface du fil sous la zone de contact pour les modèles avec différents coefficients de frottement et différents cas de charge cyclique.

Pour le cas $\mu=0, \Delta \sigma / 2=100 \mathrm{MPa}$ (Fig. 18), le critère de Crossland est maximal sur les bords de la zone de contact sans être exactement à l'interface zone de contact/zone sans contact. De plus, la zone potentielle d'initiation de fissure ne se résume pas à un point mais à un domaine d'une largeur d'environ $20 \mu \mathrm{m}$, ceci est dû au déplacement relatif entre les deux fils provoqué par l'application d'une variation de contrainte au fil inférieur.

Pour le cas $\mu=0,3, \Delta \sigma / 2=100 \mathrm{MPa}$ (Fig. 19), on distingue deux régimes à l'intérieur de la zone de contact : une zone de glissement et une zone d'adhérence, et la zone d'initiation potentielle des fissures est très proche de l'interface zones de glissement/d'adhérence.

Ces résultats sont en accord avec les observations métallographiques réalisées après les essais puisque les 


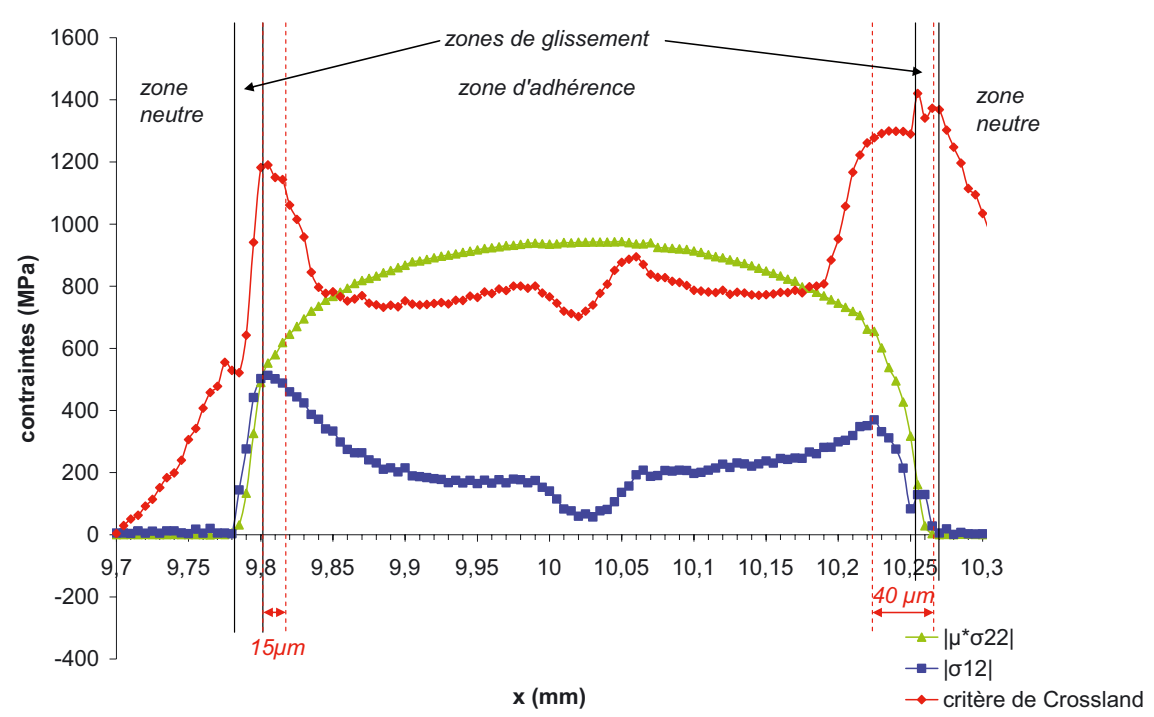

Fig. 19. Vérification du critère de Crossland à la surface du fil pour $\mu=0,3$ et $\Delta \sigma / 2=100 \mathrm{MPa}$, sous charge.

zones de contact comportent bien une zone d'adhérence et une zone de glissement, et les fissures de fretting-fatigue apparaissent sur les bords des contacts [13].

\subsection{Moyens de protection}

La politique de maintenance des ouvrages conduit à protéger les haubans contre l'usure et la corrosion. Ces protections doivent garantir une durée de vie suffisante au hauban avant qu'un remplacement ne soit nécessaire.

Les préconisations actuelles, reprises dans un guide édité par le SETRA [19], prévoient que la protection anticorrosion des torons des haubans doit être assurée de façon redondante par deux barrières complémentaires :

- une barrière interne qui doit protéger chaque toron séparément, il s'agit en général d'un revêtement métallique de type galvanisation (Fig. 20). Ce revêtement joue un rôle protecteur par un effet de barrière physique et en servant d'anode sacrificielle en cas de contact acier-eau [20]. Cette barrière interne peut également être une gaine en PEHD ou de la graisse;

- une barrière externe qui doit protéger l'ensemble du câble et qui comprend une enceinte externe et un milieu intermédiaire. L'enceinte externe doit être parfaitement étanche sur toute la longueur du câble et est en général une gaine en matière plastique (par exemple, en PEHD); sur des ouvrages existants, il peut s'agir d'une barrière type peinture, ce qui nécessite un entretien régulier pour palier les dégradations liées à l'environnement (UV, eau, ...). Le milieu intermédiaire doit empêcher la circulation d'humidité ou d'eau au contact de la première barrière, ce milieu peut être un produit de remplissage de type cire, graisse, résine, ou un courant d'air à hygrométrie contrôlée permanent autour des torons.

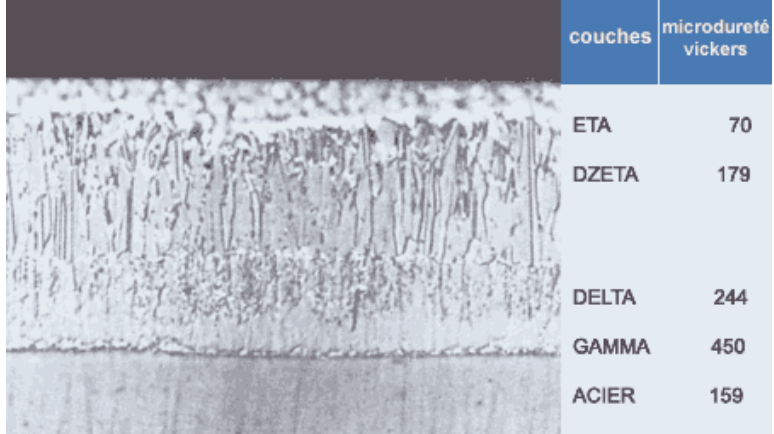

Fig. 20. Morphologie typique du revêtement de zinc sur acier [18].

Ces préconisations, tout comme les usages antérieurs, sont très orientés vers la protection anti-corrosion. Il arrive que les effets isolants et lubrifiants des différents matériaux utilisés soient en fait utiles aussi bien pour protéger le câble de la corrosion que du fretting-fatigue.

\section{Conclusions}

Les câbles utilisés en génie civil, et notamment pour les ponts à haubans et les ponts suspendus, doivent faire face à deux problématiques principales quant à leur durabilité : la tenue à la fatigue et la tenue à la corrosion.

Chacun de ces deux phénomènes est maintenant relativement bien approché individuellement :

- les différents types de corrosion, leur manifestation et leur conséquence sont connus ;

- la tenue en fatigue des câbles, le rôle prédominant de la fatigue de contact interfilaire, ainsi que les conditions d'endurance ont fait l'objet de travaux fructueux ces dernières années.

Ces travaux donnent une meilleure compréhension des phénomènes dans le cadre d'une géométrie complexe, 
et permettent leur reproduction et l'établissement de critères d'endurance.

Les dernières avancées ont mis en évidence l'effet bénéfique des dispositions prises en matière d'anticorrosion (lubrification, revêtement) sur la tenue en fatigue de ces câbles. Il reste un pas important à franchir dans la compréhension vis-à-vis de l'interaction entre les deux phénomènes fatigue et corrosion, qui fait l'objet de travaux actuels. En effet, la nature ne dissocie pas toujours ces deux pathologies sur nos ouvrages.

\section{Références}

[1] C.R. Chaplin, Failure mechanisms in wire ropes, Engineering Failure Analysis 2 (1995) 45-57

[2] A.G. Thyssen Draht, Open Spiral Strands, Full-Locked Coil Ropes, 1991

[3] K. Becker, La résistance à la fatigue des câbles d'acier, Le tréfilé 29-5 (1979) 212-215

[4] P. Brevet, F. Olivié, J.P. Guilbaud, A. Raharinaïvo, Microstructure et propriétés mécaniques des aciers pour câbles, I. Plasticité et endommagement, Bulletin des Laboratoires des Ponts et Chaussées, $\mathrm{n}^{\circ} 249$, 2004, pp. $35-48$

[5] J.M. Walton, Developments in steel cables, J. Constructional Steel Res. 39 (1996) 3-29

[6] G.A. Costello, Theory of wire rope, Springer Verlag, 1990

[7] X.H. Nguyen, Sur une modélisation du comportement mécanique d'un câble soumis à des sollicitations combinées de traction et de torsion, Thèse de doctorat, Université Joseph Fourier, Grenoble, 1994

[8] A. Nawrocki, Contribution à la modélisation des câbles monotorons par éléments finis, Thèse de doctorat, École Doctorale Sciences pour l'Ingénieur de Nantes, 1997
[9] K. Gabriel, On the fatigue strength of wires in spiral ropes, J. Energy Resources Technology 107 (1985) 107112

[10] M. Arend, The load transfer mechanisms anchoring highstrength tension elements, OIPEEC Round table, Delft, 1993

[11] SETRA-LCPC, Les ponts suspendus en France, édition LCPC, 1989

[12] R. Walther, B. Houriet, W. Isler, P. Moïa, Ponts haubanés, Presses Polytechniques Romandes, 1985

[13] D. Siegert, Mécanismes de fatigue de contact dans les câbles de haubanage du Génie Civil, Thèse de doctorat, École Centrale de Nantes, Université de Nantes, 1997

[14] L. Dieng, J.R. Urvoy, D. Siegert, P. Brevet, V. Perier, C. Tessier, Assessment of lubrication and zinc coating on the high cycle fretting fatigue behaviour of high strength steel wires, OIPEEC, Johannesburg, 2007, pp. 85-97

[15] J.R. Urvoy, D. Siegert, L. Dieng, P. Brevet, Influence des revêtements métalliques et des lubrifiants sur la fatigue des contacts interfilaires de câbles, $17^{\mathrm{e}}$ Congrès Français Mécanique, Troyes, 2005

[16] V. Périer, L. Dieng, L. Gaillet, Modélisation de la fatigue de contact dans les câbles corrodés, $18^{\mathrm{e}}$ Congrès Français Mécanique, Grenoble, 2007

[17] B. Crossland, Effect of large hydrostatic pressure on the torsional fatigue strengh of an alloy steel, International Conference on Fatigue of Metals, Londres, 1959, pp. 138149

[18] www.galvazinc.com

[19] SETRA, Haubans - Recommandations de la commission interministérielle de la précontrainte, 2001

[20] Norme NF A91-131 (1962-04-01), Fils d'acier galvanisés à chaud, Spécification du revêtement de zinc, 1962 